\title{
Probing the Origin of Neutrino Masses and Mixings via Doubly Charged Scalars: Complementarity of the Intensity and the Energy Frontiers
}

\author{
Tanja Geib, ${ }^{1}$ Stephen F. King, ${ }^{2}$ Alexander Merle,,${ }^{1,2}$ Jose Miguel No,${ }^{3}$ and Luca Panizzi ${ }^{2}$ \\ ${ }^{1}$ Max-Planck-Institut für Physik (Werner-Heisenberg-Institut), Föhringer Ring 6, 80805 München, Germany \\ ${ }^{2}$ Physics and Astronomy, University of Southampton, Southampton, SO17 1BJ, United Kingdom \\ ${ }^{3}$ Department of Physics and Astronomy, University of Sussex, Brighton BN1 9QH, United Kingdom
}

(Dated: May 23, 2016)

\begin{abstract}
We discuss how the intensity and the energy frontiers provide complementary constraints within a minimal model of neutrino mass involving just one new field beyond the Standard Model at accessible energy, namely a doubly charged scalar $S^{++}$and its antiparticle $S^{--}$. In particular we focus on the complementarity between high-energy LHC searches and low-energy probes such as lepton flavor violation. Our setting is a prime example of how high- and low-energy physics can cross-fertilize each other.
\end{abstract}

The origin of neutrino mass and mixing is an outstanding open question, as the existence of massive neutrinos, which follows from the discovery of neutrino oscillations [1, 2], cannot be accommodated within the Standard Model (SM) of particle physics. Together with other puzzles like the nature of Dark Matter or the generation of the observed matter-antimatter asymmetry in the Universe, this constitutes a leading motivation to search for new physics beyond the SM.

The experimental effort to unravel the nature and properties of such new physics is pursued along three main avenues: the energy, intensity, and cosmic frontiers, which provide highly complementary probes of new physics. A prime example of such a complementarity arises if the new physics responsible for neutrino masses and mixings lies not very far above the electroweak scale, case in which both low and high-energy experiments could be sensitive to it. However, even though there are well-motivated scenarios for the generation of neutrino masses and mixings which predict signatures at both the intensity and high-energy frontiers, such as low-scale seesaw models (see e.g. the discussion in [3]) and loopinduced models 4 9, these scenarios generically predict the existence of multiple new particles, making concrete predictions for phenomenology difficult to extract.

A doubly charged scalar particle $S^{++}$is predicted in a large class of these scenarios in connection to Lepton Number Violation (LNV) and the generation of neutrino masses. An extension of the SM by just one new particle at accessible energy, $S^{++}$(being $S U(2)_{L}$ singlet to avoid the introduction of extra degrees of freedom from an $S U(2)_{L}$ multiplet), and in the presence of effective operators giving rise to LNV, provides the most minimal framework which captures the main features of a large class of neutrino mass models [10. It allows to fully exploit the complementarity between collider searches and low-energy probes such as lepton flavor violating (LFV) processes.

In this work we manifestly explore the complementarity of the two experimental avenues as a probe of a doubly charged (but $S U(2)_{L}$ singlet) scalar particle $S^{++}$. From the low-energy perspective (intensity frontier), while the experimental limits on the LFV processes $\mu \rightarrow e \gamma$ from MEG [11] and $\mu \rightarrow 3 e$ from SINDRUM [12] are at present the most stringent ones, the most dramatic upcoming experimental advances are to occur in $\mu^{-}-e^{-}$conversion in nuclei, which is expected to become the most sensitive LFV probe in the future 13], expected to reach a sensitivity to branching ratios of $10^{-17}$ already in the nearer future 14. At the same time, the Large Hadron Collider (LHC) will probe during Run-II the TeV region for new particles like $S^{++}$. It is these two probes that we concentrate on here, assessing their respective reach in these scenarios. Our discussion reveals how strongly information from both the intensity and energy frontiers can complement each other, to maximize our benefit from on-going and near-future experiments searching for new physics.

\section{INTENSITY/ENERGY COMPLEMENTARITY: A KEY APPROACH TO NEUTRINO MASSES}

Let us start by discussing the theoretical framework for the SM with the addition of an $S U(2)_{L}$ singlet, doubly charged scalar $S \equiv S^{++}$or $S^{--}$. Our renormalizable Lagrangian is

$$
\mathcal{L}=\mathcal{L}_{\mathrm{SM}}+\left(D_{\mu} S^{++}\right)^{\dagger}\left(D^{\mu} S^{++}\right)+f_{a b} \overline{\left(\ell_{R}\right)_{a}^{c}} \ell_{R b} S^{++}+\text {h.c. },
$$

with $a, b=e, \mu, \tau$ being flavor indices and $f_{a b}$ a symmetric matrix in flavor space. The scalar $S^{++}$is assumed to have a mass $M_{S}$. The Lagrangian (1) conserves lepton number, thus not leading to neutrino mass generation. However, allowing for non-renormalizable operators containing both $S^{++}$and SM fields leads to LNV. Assuming that $S^{++}$is connected to the generation of neutrino masses (which by construction forbids the $D=5$ Weinberg operator), the leading LNV operator appears at $D=7$ [10] (see also [15, 16]):

$$
\frac{\xi}{\Lambda^{3}}\left[H^{T} i \sigma_{2}\left(D_{\mu} H\right)\right]\left[H^{T} i \sigma_{2}\left(D^{\mu} H\right)\right] S^{++}+\text {h.c. }
$$




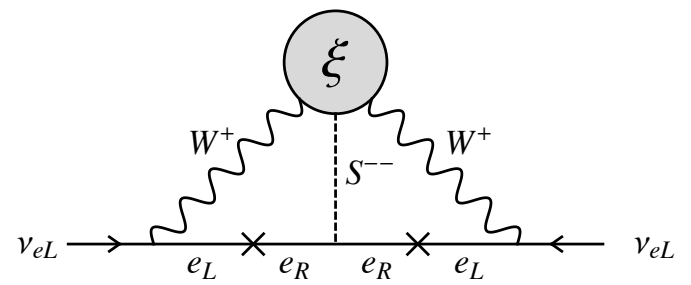

FIG. 1. Neutrino mass generation for the example of the $m_{e e}^{\nu}$ element of the full neutrino mass matrix. Similar diagrams are responsible for the full matrix $m_{a b}^{\nu}$, where $a, b=e, \mu, \tau$.

which leads to an interaction $S^{ \pm \pm} W^{\mp} W^{\mp}$. Combined with the last term in (1), this breaks lepton number by two units and at two-loop order gives rise to light neutrino masses of Majorana nature, as shown in Figure 1 . by adding only one new particle to the SM.

We stress that the interactions in (1) suffice to describe the physical processes which allow to fully exploit the low-high energy complementarity in this class of scenarios. Nevertheless, the key for complementarity is that the matrix $f_{a b}$ is far from arbitrary, as it enters into the generation of the neutrino mass matrix $\left(m_{a b}^{\nu}\right)$ :

$$
\left(m_{a b}^{\nu}\right) \sim\left(\begin{array}{ccc}
m_{e}^{2} f_{e e} & m_{e} m_{\mu} f_{e \mu} & m_{e} m_{\tau} f_{e \tau} \\
m_{e} m_{\mu} f_{e \mu} & m_{\mu}^{2} f_{\mu \mu} & m_{\mu} m_{\tau} f_{\mu \tau} \\
m_{e} m_{\tau} f_{e \tau} & m_{\mu} m_{\tau} f_{\mu \tau} & m_{\tau}^{2} f_{\tau \tau}
\end{array}\right),
$$

with the symmetric nature of $\left(m_{a b}^{\nu}\right)$ being just a reflection of the light neutrinos being Majorana particles. The structure of $\left(m_{a b}^{\nu}\right)$ is constrained by the measurements of all light neutrino mass squared differences and leptonic mixing angles by neutrino oscillation experiments [17, 18. Combining these with current bounds on $f_{a b}$ and $M_{S}$ from LFV processes like $\mu \rightarrow e \gamma$ [1921 and the LNV neutrinoless double $\beta$-decay process, we extract three representative average sets of couplings $\left\{f_{a b}\right\}$ [10, called red $\left(f_{e e} \simeq f_{e \tau} \simeq 0\right)$, purple $\left(f_{e e} \simeq 0 \&\right.$ $\left.\left|f_{e \mu} f_{\mu \mu}^{*}\right| \simeq\left|f_{\mu \tau}^{*} f_{e \tau}\right|\right)$, and blue (only $\left.\left|f_{e \mu} f_{\mu \mu}^{*}\right| \simeq\left|f_{\mu \tau}^{*} f_{e \tau}\right|\right)$ in Tab. I.

\begin{tabular}{c|c|c|c|c|c|c} 
& $f_{e e}$ & $f_{e \mu}$ & $f_{e \tau}$ & $f_{\mu \mu}$ & $f_{\mu \tau}$ & $f_{\tau \tau}$ \\
\hline red & $<10^{-10}$ & $10^{-2}$ & $<10^{-10}$ & $10^{-4}$ & $10^{-5}$ & $10^{-6}$ \\
\hline purple & $<10^{-10}$ & $10^{-3}$ & $10^{-2}$ & $10^{-3}$ & $10^{-4}$ & $10^{-5}$ \\
\hline blue & $10^{-1}$ & $10^{-4}$ & $10^{-2}$ & $10^{-3}$ & $10^{-4}$ & $10^{-5}$ \\
\hline
\end{tabular}

TABLE I. Benchmark sets of couplings for the possible neutrino mass scenarios.

This is the ideal situation from the point of view of complementarity between LHC searches and low-energy LFV probes, from which $\mu^{-}-e^{-}$conversion in nuclei is going to be the most powerful in the upcoming future. For each set of couplings (red, purple and blue) the only free parameter in both cases is the mass of the new particle $S^{++}$. In the following we discuss the analyses for both, and the resulting complementarity, for both the benchmark slopes (displayed as red, purple and blue lines corresponding to the representative sets of couplings in Tab. I) and for benchmark points (displayed as red, purple, and blue dots) taken from [10, where all slopes and points correctly describe the neutrino masses and mixings.

\section{Intensity Frontier: $\mu^{-}-e^{-}$Conversion in Nuclei}

We now explore the process of $\mu^{-}-e^{-}$conversion on a nucleus [22, 23] in the presence of $S^{++}$and its antiparticle $S^{--}$. We discuss here the main results and present the technical details of the calculation elsewhere [24]. The branching fraction of $\mu-e$ conversion with respect to ordinary muon capture rate $\Gamma_{\text {Capt }}$, in the limit that the long-range contributions (mediated by the photon $\gamma$ in Figure 22 dominate the process, can be written as:

$$
\mathrm{BR}\left(\mu^{-} N \rightarrow e^{-} N\right) \simeq \frac{8 \alpha_{\mathrm{EM}}^{5} m_{\mu} Z_{\mathrm{eff}}^{4} F_{p}^{2}}{\Gamma_{\mathrm{Capt}}} \Xi_{\text {particle }}^{2},
$$

with the effective atomic charge $Z_{\text {eff }}$ and the nuclear matrix element $F_{p}$. The term $\Xi_{\text {particle encodes the parti- }}$ cle physics part of the amplitude. The above factorization of the branching fraction into particle and nuclear physics parts is made possible precisely by neglecting the short-range (non-photonic) contributions to the amplitude (Figure 2, Right), which are found to be parametrically suppressed by roughly $\mathcal{O}\left(m_{\mu}^{2} / M_{W}^{2}\right) \sim 10^{-5}[24$. This factorization is very convenient, since all nuclear physics uncertainties and isotopic dependences can be absorbed into the experimental bounds. The particle physics amplitude $\Xi_{\text {particle }}$ is given by [24]:

$$
\begin{aligned}
& \Xi_{\text {particle }}=\frac{\left|\Pi_{e} f_{e e}^{*} f_{e \mu}+\Pi_{\mu} f_{e \mu}^{*} f_{\mu \mu}+\Pi_{\tau} f_{e \tau}^{*} f_{\tau \mu}\right|}{12 \sqrt{2} \pi^{2} m_{\mu} M_{S}^{2}} \\
& \text { with } \Pi_{a}=4 m_{a}^{2} m_{\mu}-m_{\mu}^{3}\left[1-\ln \left(m_{a}^{2} / M_{S}^{2}\right)\right] \\
& +2\left(m_{\mu}^{2}-2 m_{a}^{2}\right) \sqrt{m_{\mu}^{2}+4 m_{a}^{2}} \operatorname{Arctanh}\left[m_{\mu} / \sqrt{m_{\mu}^{2}+4 m_{a}^{2}}\right] .
\end{aligned}
$$

The structure of $\mu-e-\gamma$ in the middle diagram of Figure 2 enters the loop-functions $\Pi_{e, \mu, \tau}$.

While naively one could expect not much to change compared to $\mu \rightarrow e \gamma$, whose amplitude is proportional to $C \cdot\left|f_{e e}^{*} f_{e \mu}+f_{e \mu}^{*} f_{\mu \mu}+f_{e \tau}^{*} f_{\tau \mu}\right| / M_{S}^{2}$ (where $C$ is a constant incorporating all numerical factors), the coefficients $\Pi_{a}(a=e, \mu, \tau)$ in (5) cannot be factored out. This immediately explains why the corresponding bound will be very strong: the benchmark scenarios described in [10] all avoid the bound from $\mu \rightarrow e \gamma$ by relying on some cancellation among the couplings in the expression $\left(f_{e e}^{*} f_{e \mu}+f_{e \mu}^{*} f_{\mu \mu}+f_{e \tau}^{*} f_{\tau \mu}\right)$. This cancellation is spoiled if there is no common prefactor $C$ anymore. Thus, when taking into account the experimental improvements in searches for $\mu^{-}-e^{-}$conversion, this process provides a 
very strong bound on the otherwise perfectly working scenarios.

The resulting bounds on the scenarios found in 10 are displayed in Figure 4. The model predictions are illustrated in two ways, for actual benchmark points (displayed as red, purple, and blue dots) taken from [10], and for the representative sets of couplings in Tab. I (displayed as red, purple, and blue lines), which comprise "averaged" versions of the points with low $M_{S}$ and illustrate how the bounds vary with the scalar mass $M_{S}$ for fixed couplings. Note that, for large $M_{S}$, the spread of the points around the line becomes bigger, which is expected from the LFV/LNV bounds generally becoming weaker for large $M_{S}$. As visible from Figure $4, \mu^{-}-e^{-}$ conversion bounds push from top to bottom. We have collected several bounds from current and future experiments 14, 25 28. The different scenarios can be constrained depending on the exact values of the model parameters. For example, the blue line is easier to constrain than the red/purple lines. The reason is that the coefficients $\Pi_{e, \mu, \tau}$, while being sufficiently different to spoil cancellations between the three contributions to the total amplitude, are nevertheless all of the same order. Thus, the benchmark lines with the largest value of $\left|f_{e e}^{*} f_{e \mu}\right|,\left|f_{e \mu}^{*} f_{\mu \mu}\right|$ or $\left|f_{e \tau}^{*} f_{\tau \mu}\right|$ will be easiest to constrain.
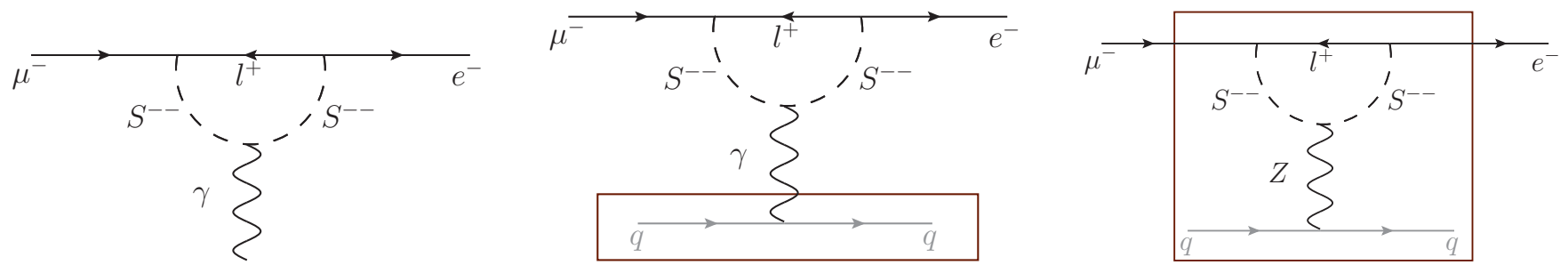

FIG. 2. Example diagrams for $\mu \rightarrow e \gamma$ (left) and $\mu$-e conversion long-range (middle) and short-range (right) contributions. The parts inside the large rectangular frames happen inside the nucleus, which necessitates the distinction of long- and short-range.

\section{Energy Frontier: LHC Searches}

Direct searches at the LHC provide a powerful probe of the existence of $S^{++}$and its antiparticle $S^{--}$, highly complementary to $\mu$-e conversion and further LFV processes. At the LHC, the dominant production mode of $S^{++}$is pair-production through the Drell-Yan (DY) process, via a $Z$-boson/photon in the s-channel, as depicted in Figure 3 (Top). Other channels, like pair-production through vector boson fusion (VBF) (see e.g. Figure 3 (Bottom) are largely subdominant, and we will not consider them in the present analysis. We however stress that upon discovery of $S^{++}$, these channels could yield valuable information on the underlying theory. Moreover, a potential Higgs portal interaction $\lambda_{S}|H|^{2}|S|^{2}$, as well as the linear interaction (2) would yield new, modeldependent avenues for probing the existence of $S^{++}$(the interplay between these and the dominant DY production will be explored elsewhere [29]). We nevertheless stress that the effect of the Higgs portal interaction $\lambda_{S}|H|^{2}|S|^{2}$ on the $h \rightarrow \gamma \gamma$ decay of the $125 \mathrm{GeV}$ Higgs is too small to be probed at the LHC for $\lambda_{S} \lesssim 1$ and/or $m_{S} \gtrsim 300$ $\mathrm{GeV}$ [29]

We now analyze the LHC sensitivity to DY production and same-sign di-lepton decays of $S^{++}$for the three possible coupling patterns described above: red, purple, and blue. We first concentrate on the LHC $7 \mathrm{TeV}$ experimental searches for doubly-charged scalars by ATLAS/CMS [30, 31]. Using the $7 \mathrm{TeV}$ Next-to-leadingorder (NLO) DY production cross-section $\sigma$ values and selection efficiencies $\epsilon_{4 \ell}, \epsilon_{3 \ell \tau}, \epsilon_{2 \ell 2 \tau}$ (with $\ell=e, \mu$, and $\tau$ denoting a hadronically decaying $\tau$-lepton) from [30, all as a function of $M_{S}$, we can easily derive the bound on $M_{S}$ that [30] yields for the red, purple, and blue benchmarks. The number of signal events for each benchmark is given by:

$$
\begin{aligned}
s & =\sigma\left(M_{S}\right) \times \mathcal{L} \\
& \times\left\{\mathrm{BR}_{\ell \ell}^{2} \epsilon_{4 \ell}+2 \mathrm{BR}_{\ell \ell} \mathrm{BR}_{\ell \tau}\left[b r_{\tau_{\ell}} \epsilon_{4 \ell}+b r_{\tau_{H}} \epsilon_{3 \ell \tau}\right]\right. \\
& \left.+\mathrm{BR}_{\ell \tau}^{2}\left[b r_{\tau_{\ell}}^{2} \epsilon_{4 \ell}+2 b r_{\tau_{H}} b r_{\tau_{\ell}} \epsilon_{3 \ell \tau}+b r_{\tau_{H}}^{2} \epsilon_{2 \ell 2 \tau}\right]\right\}
\end{aligned}
$$

where $\mathrm{BR}_{\ell \ell}$ and $\mathrm{BR}_{\ell \tau}$ are, respectively, the $S^{++}$branching fractions into two light leptons $(\ell=e, \mu)$ and into a light lepton and a $\tau$-lepton, which can be directly obtained as ratios of squared couplings from Tab. I The hadronic and leptonic branching fractions of a $\tau$-lepton are respectively given by $b r_{\tau_{H}} \simeq 0.65, b r_{\tau_{\ell}} \simeq 0.35$.

The $M_{S}$ limit is then obtained using the $\mathrm{CL}_{s}$ procedure [32, 33, for which we construct the likelihood ratio test-statistics $Q$, corresponding to the ratio of likelihoods for the signal+background $(s+b)$ and background only (b) hypotheses for the observed number of events in each experimental bin $n_{i}$, and then compute the exclusion con- 


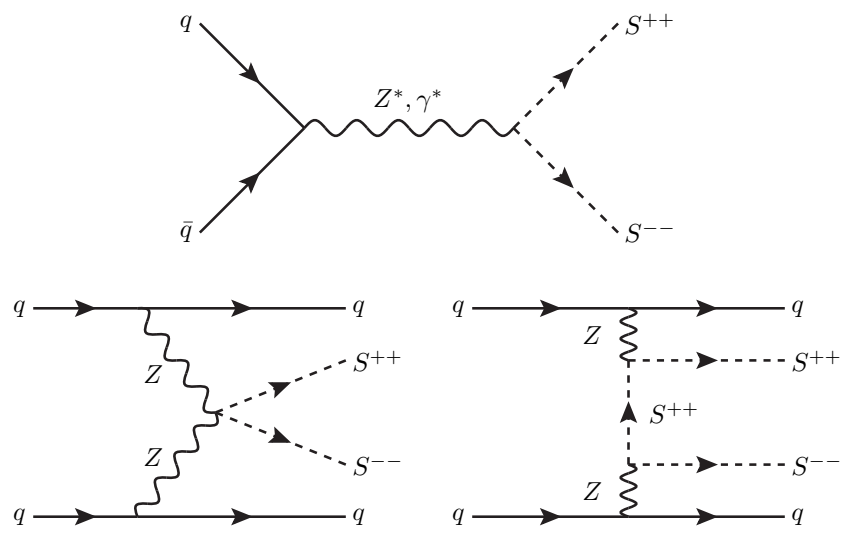

FIG. 3. $S^{++}$production channels at the LHC: Drell-Yan (Top); Vector Boson Fusion (Bottom).

\begin{tabular}{cc|c|ccc}
\hline $\begin{array}{c}\text { Energy } \\
(\mathrm{TeV})\end{array}$ & $\begin{array}{c}\mathcal{L} \\
(\mathrm{fb})\end{array}$ & $\begin{array}{c}\text { Ideal } \\
\text { Bound }\end{array}$ & $\begin{array}{c}\text { red } \\
\mathrm{BR}_{\ell \ell}=1\end{array}$ & $\begin{array}{c}\text { purple } \\
\mathrm{BR}_{\ell \tau}=0.98\end{array}$ & $\begin{array}{c}\text { blue } \\
\mathrm{BR}_{\ell \ell}=0.99\end{array}$ \\
\hline 7 & 4.9 & 423 & 364 & 293 & 363 \\
\hline \multirow{2}{*}{13} & 100 & 900 & 781 & 664 & 780 \\
& 300 & 1102 & 977 & 811 & 976 \\
\hline
\end{tabular}

TABLE II. LHC $M_{S}$ bounds (in GeV) for the different benchmarks. The ideal bound assumes $100 \%$ efficiencies for all channels $\left(\epsilon_{4 \ell}, \epsilon_{3 \ell \tau}, \epsilon_{2 \ell 2 \tau}=1\right)$. fidence level of the signal under the assumption of the $b$ hypothesis by comparing the $p$-values of the Poissonian distributions of the $s+b$ and $b$ hypotheses:

$$
\mathrm{CL}_{s}=\frac{1-p(s+b)}{1-p(b)} \quad, \quad Q=e^{-s} \prod_{i=1}^{N_{\mathrm{bins}}}\left(1+\frac{s_{i}}{b_{i}}\right)^{n_{i}}
$$

For the LHC $13 \mathrm{TeV}$ expected limits, we have computed the Leading Order DY pair-production cross sections with MadGraph5_aMC@NLO 34] and obtained the rescaled NLO cross-sections via an average, $M_{S^{-}}$ independent, $\kappa$-factor of 1.25 [35. We then consider both an ideal scenario, with $100 \%$ signal selection efficiencies, and a conservative one in which we extrapolate to LHC $13 \mathrm{TeV}$ the values for the efficiencies $\epsilon_{4 \ell}, \epsilon_{3 \ell \tau}, \epsilon_{2 \ell 2 \tau}$ at $7 \mathrm{TeV}$ (which are expected to improve from LHC Run 1 to Run 2). In both cases, we use (6) and the $\mathrm{CL}_{s}$ method under the hypothesis of no background events (and therefore no observed events). To obtain a 95\% CL exclusion under these hypotheses, the number of signal events must be larger than 3 . The results for the $M_{S}$ bounds at both $7 \mathrm{TeV}$ and $13 \mathrm{TeV}$ are shown in Tab. III and then included in Figure 4, which clearly shows the complementarity between LFV and LHC searches for the three benchmarks.
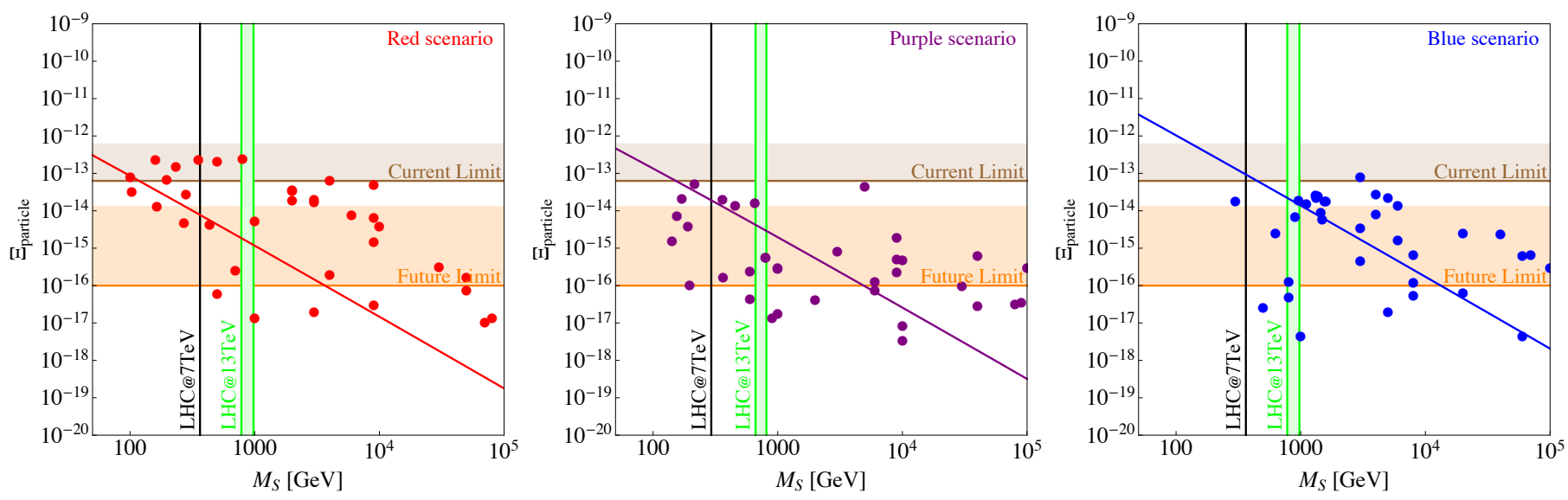

FIG. 4. Particle physics parts $\Xi_{\text {particle }}$ as functions of the scalar mass $M_{S}$ for the red/purple/blue scenarios. Different limits from $\mu^{-}-e^{-}$conversion and from LHC searches are indicated, see text for details.

\section{CONCLUSION}

We have shown how the intensity and the energy frontiers provide complementary constraints within a minimal model of neutrino mass and mixing involving just one new particle beyond the Standard Model, namely a doubly charged scalar $S^{++}$and its antiparticle $S^{--}$. Focussing on the complementarity between LHC searches and low energy probes such as lepton flavor violation, the results are summarised in Figure 4. The complementary nature of these approaches is very clear from this figure, with the LHC able to exclude scalar masses approaching $1 \mathrm{TeV}$, while $\mu^{-}-e^{-}$conversion holds the promise of orders of magnitude improvement in constraining the particle physics amplitude in (5), albeit with rather large uncertainties due to nuclear physics.

From a general perspective, the considered framework provides a minimal and clear example of complementarity between two of the most important experimental particle 
physics strategies presently being pursued, towards uncovering the physics beyond the Standard Model, which must necessarily be present to account for neutrino mass and mixing.

Note added: As this paper was being submitted, 36 appeared, which also discusses the importance of the complementarity of high- and low-energy data for the case of doubly charged scalars. While that paper also discusses several LFV aspects, its main focus is the muon magnetic dipole moment $(g-2)$, while our paper focuses strongly on the complementarity between LHC direct searches and $\mu-e$ conversion.

Acknowledgements We are indebted to Richard Ruiz for very useful comments on a first version of this work. SFK acknowledges partial support from the STFC Consolidated ST/J000396/1 grant. AM acknowledges warm hospitality by the University of Southampton, where part of this work has been performed, as well as partial support by the Micron Technology Foundation, Inc. SFK and AM furthermore acknowledge partial support by the European Union FP7 ITN-INVISIBLES (Marie Curie Actions, PITN-GA-2011-289442). JMN is supported by the People Programme (Marie curie Actions) of the European Union Seventh Framework Programme (FP7/2007-2013) under REA grant agreement PIEF-GA2013-625809.

[1] Y. Fukuda et al. (Super-Kamiokande), Phys. Rev. Lett. 81, 1562 (1998), hep-ex/9807003.

[2] Q. R. Ahmad et al. (SNO), Phys. Rev. Lett. 89, 011301 (2002), nucl-ex/0204008.

[3] R. N. Mohapatra et al., Rept. Prog. Phys. 70, 1757 (2007), hep-ph/0510213.

[4] A. Zee, Phys. Lett. B93, 389 (1980), [Erratum: Phys. Lett.B95,461(1980)].

[5] K. S. Babu, Phys. Lett. B203, 132 (1988).

[6] L. M. Krauss, S. Nasri, and M. Trodden, Phys. Rev. D67, 085002 (2003), hep-ph/0210389.

[7] E. Ma, Phys. Rev. D73, 077301 (2006), hep-ph/0601225.

[8] M. Aoki, S. Kanemura, and O. Seto, Phys. Rev. Lett. 102, 051805 (2009), 0807.0361.

[9] M. Gustafsson, J. M. No, and M. A. Rivera, Phys. Rev. Lett. 110(21), 211802 (2013), [Erratum: Phys. Rev. Lett. 112, no.25, 259902 (2014)], 1212.4806.

[10] S. F. King, A. Merle, and L. Panizzi, JHEP 11, 124 (2014), 1406.4137.

[11] J. Adam et al. (MEG), Phys. Rev. Lett. 110, 201801
(2013), 1303.0754.

[12] U. Bellgardt et al. (SINDRUM), Nucl. Phys. B299, 1 (1988).

[13] M. Raidal et al., Eur. Phys. J. C57, 13 (2008), 0801.1826.

[14] Y. G. Cui et al. (COMET), Report No. J-PARC P21 (2009).

[15] F. del Aguila, A. Aparici, S. Bhattacharya, A. Santamaria, and J. Wudka, JHEP 1206, 146 (2012), 1204.5986.

[16] M. Gustafsson, J. M. No, and M. A. Rivera, Phys. Rev. D90, 013012 (2014), 1402.0515.

[17] M. C. Gonzalez-Garcia, M. Maltoni, and T. Schwetz, JHEP 11, 052 (2014), 1409.5439.

[18] J. Bergstrom, M. C. Gonzalez-Garcia, M. Maltoni, and T. Schwetz, JHEP 09, 200 (2015), 1507.04366.

[19] B. W. Lee and R. E. Shrock, Phys. Rev. D16, 1444 (1977).

[20] T. P. Cheng and L.-F. Li, Phys. Rev. Lett. 45, 1908 (1980).

[21] L. Lavoura, Eur. Phys. J. C29, 191 (2003), hep$\mathrm{ph} / 0302221$.

[22] S. Weinberg and G. Feinberg, Phys. Rev. Lett. 3, 111 (1959).

[23] W. J. Marciano and A. I. Sanda, Phys. Rev. Lett. 38, 1512 (1977).

[24] T. Geib and A. Merle (2015), accepted by Phys. Rev. D, 1512.04225 .

[25] C. Dohmen et al. (SINDRUM II), Phys. Lett. B317, 631 (1993).

[26] W. Honecker et al. (SINDRUM II), Phys. Rev. Lett. 76, 200 (1996).

[27] R. K. Kutschke, in Proceedings, 31st International Conference on Physics in collisions (PIC 2011) (2011), 1112.0242, URL http://inspirehep.net/record/ 1079590/files/arXiv:1112.0242.pdf

[28] R. J. Barlow, Nucl. Phys. Proc. Suppl. 218, 44 (2011).

[29] J. M. No and L. Panizzi, Work in Progress.

[30] S. Chatrchyan et al. (CMS), Eur. Phys. J. C72, 2189 (2012), 1207.2666.

[31] G. Aad et al. (ATLAS), Eur. Phys. J. C72, 2244 (2012), 1210.5070 .

[32] A. L. Read, in Workshop on confidence limits, CERN, Geneva, Switzerland, 17-18 Jan 2000: Proceedings (2000), URL http://weblib.cern.ch/abstract? CERN-OPEN-2000-205

[33] A. L. Read, J. Phys. G28, 2693 (2002), [,11(2002)].

[34] J. Alwall, R. Frederix, S. Frixione, V. Hirschi, F. Maltoni, O. Mattelaer, H. S. Shao, T. Stelzer, P. Torrielli, and M. Zaro, JHEP 07, 079 (2014), 1405.0301.

[35] M. Muhlleitner and M. Spira, Phys. Rev. D68, 117701 (2003), hep-ph/0305288.

[36] J. Chakrabortty, P. Ghosh, S. Mondal, and T. Srivastava (2015), 1512.03581. 